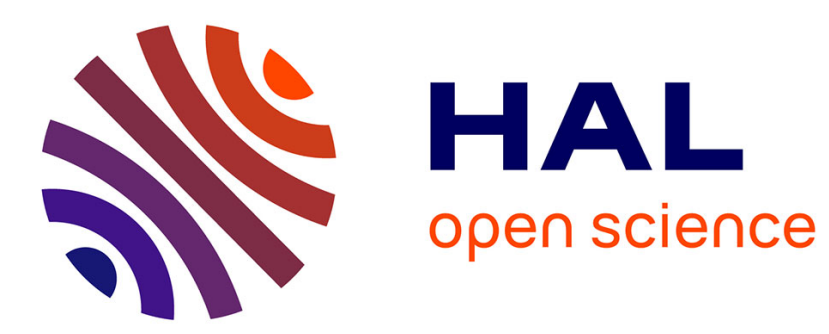

\title{
Grey-level hit-or-miss transforms-Part II: Application to angiographic image processing
}

Benoît Naegel, Nicolas Passat, Christian Ronse

\section{To cite this version:}

Benoît Naegel, Nicolas Passat, Christian Ronse. Grey-level hit-or-miss transforms-Part II: Application to angiographic image processing. Pattern Recognition, 2007, 40 (2), pp.648-658. 10.1016/j.patcog.2006.06.011 . hal-01694417

HAL Id: hal-01694417

\section{https://hal.univ-reims.fr/hal-01694417}

Submitted on 26 Feb 2018

HAL is a multi-disciplinary open access archive for the deposit and dissemination of scientific research documents, whether they are published or not. The documents may come from teaching and research institutions in France or abroad, or from public or private research centers.
L'archive ouverte pluridisciplinaire HAL, est destinée au dépôt et à la diffusion de documents scientifiques de niveau recherche, publiés ou non, émanant des établissements d'enseignement et de recherche français ou étrangers, des laboratoires publics ou privés. 


\title{
Grey-level hit-or-miss transforms - Part II: Application to angiographic image processing
}

\author{
Benoît Naegel $^{\mathrm{a}, *}$, Nicolas Passat $^{\mathrm{b}, \mathrm{c}}$, Christian Ronse $^{\mathrm{b}}$ \\ ${ }^{a}$ EIG-HES (École d'Ingénieurs de Genève), 4 rue de la Prairie, CH-1202 Genève, Switzerland \\ ${ }^{\mathrm{b}}$ LSIIT, UMR 7005 CNRS-ULP (Laboratoire des Sciences de l'Image, de l'Informatique et de la Télédétection), Bd S. Brant, BP 10413, \\ F-67412 Illkirch Cedex, France \\ ${ }^{\mathrm{c}}$ Institut Gaspard Monge, Laboratoire A2SI (Algorithmique et Architecture des Systèmes Informatiques), Groupe ESIEE, Cité Descartes, \\ BP 99, F-93162 Noisy-le-Grand Cedex, France
}

\begin{abstract}
The hit-or-miss transform (HMT) is a fundamental operation on binary images, widely used since forty years. As it is not increasing, its extension to grey-level images is not straightforward, and very few authors have considered it. Moreover, despite its potential usefulness, very few applications of the grey-level HMT have been proposed until now. Part I of this paper [1] was devoted to the description of a theory enabling to a unify the main definitions of the grey-level HMT, mainly proposed by Ronse and Soille, respectively. Part II of this paper, developed hereafter, deals with the applicative potential of the grey-level HMT, illustrated by its use for vessel segmentation from 3D angiographic data. Different HMT-based segmentation methods are then described and analysed, leading to concrete analysis techniques for brain and liver vessels, but also providing algorithmic strategies which could further be used for many other kinds of image processing applications.
\end{abstract}

Key words: Mathematical morphology, hit-or-miss transform, grey-level interval operator, angiographic image processing.

\section{Introduction: 3D angiographic imaging}

The development of medical imaging techniques during the last twenty years, has led to the creation of three-dimensional (3D) data acquisition processes. The most important ones are magnetic resonance imaging (MRI) and computed tomography (CT), which are respectively based on nuclear magnetic resonance properties of the atoms and on their behaviour when exposed to X-rays.

\footnotetext{
* Corresponding author: Benoît Naegel. Email: benoit.naegel@hesge.ch, tel: (+41) (0)22 3380566 .
}

Since visualisation of vessels or flowing blood (for surgery planning, vascular pathology detection or functional analysis) constitutes an important issue in medical imaging, both CT and MRI have been adapted to enable a correct discrimination of vascular structures. These specific techniques, called computed tomography angiography (CTA) [2] and magnetic resonance angiography (MRA) [3] generally provide 3D data of vessels (i.e., of blood) with a millimetric or submillimetric resolution. It has to be noticed that CTscans of hepatic structures, which require an injection of a contrast material in order to discriminate healthy from non healthy parts of the liver, also provide information on the vascular network. Examples of slices 

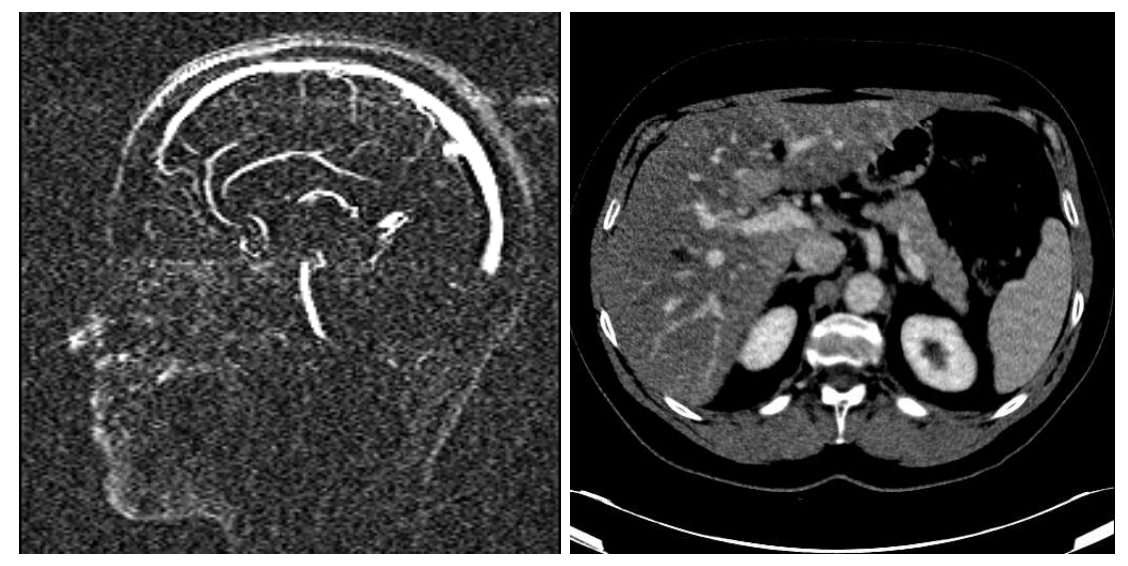

Fig. 1. Slices of three-dimensional angiographic data. Left: sagittal slice (medial plane) of a MRA of the brain, visualising arterial and venous flowing blood. Right: axial slice of a CT-scan of the abdomen, visualising hepatic structures and branches of the portal network (on the left part of the slice).

of MRA and liver CT-scans are illustrated in Fig. 1.

Angiographic images are generally characterized by a high signal of the flowing blood (obtained by injecting a contrast agent: gadolinium in contrast-enhanced MRA [4] or iodinated contrast material in CT-scan, or by only using the physical properties linked to the blood flow in phase-contrast [5] and time-of-flight [6] MRA) by comparison to other tissues, whose signal is generally lower or removed.

This signal contrast between blood and other tissues, coupled with geometric and topological properties of blood vessels (which are elongated structures generally organized in tree-like networks) theoretically enable to easily analyse $3 \mathrm{D}$ angiographic data. However, such data are generally huge, being composed of hundreds of slices, and often present a low SNR. A straightforward consequence of these properties is to make three-dimensional angiographic data analysis a long and error prone task for radiologists.

\section{Use of grey-level HMT for vessel segmentation}

Although classical two-dimensional visualisation techniques such as maximum intensity projection (MIP) [7] are often used for the analysis of 3D angiographies, the development of vessel segmentation strategies enabling to generate a volumic object corresponding to the vascular network represented in a CTA or a MRA, has been an active research field for the last fifteen years. Several papers have addressed the problem of 3D vessel segmentation, some of them being cited and discussed in the following overviews [8-11]. Most of the proposed methods rely on assumptions concerning the high intensity of blood signal and the tubular shape of vessels. These assumptions have been used to design algorithms based on many image processing concepts including deformable models, vessel-tracking or mathematical morphology. However, the use of grey-level HMT had never been considered before our work proposed in [12-15] (more generally, grey-level HMT had not been much considered for image processing applications, before this work, except by Soille in [16], and by Barat et al. who used morphological probing for a few industrial applications [17,18], or illustrated its behaviour when applied on 2D medical data [19]).

Despite its low involvement in 3D vessel segmentation, the grey-level HMT presents properties justifying a more intensive use. Indeed, its definition in terms of foreground and background structuring elements (SE) is appropriate to the invariant vessel properties in terms of shape and intensity with respect to the remaining tissues. For vessel segmentation purpose, the HMT can be used in different ways. It can be involved in classical filtering strategies consisting in applying well-chosen structuring elements on the whole image, in order to detect the vessels. It can also be used as a part of heuristic criteria providing information on the "vesselness" of voxels or sets of voxels of the pro- 
cessed image, such kinds of criteria being adapted to be incorporated in vessel-tracking or region-growing segmentation strategies.

The use of grey-level HMT for vessel segmentation from 3D angiographic data however requires to correctly choose parameters such as shape or intensity, and to deal with the possibly high algorithmic complexity inherent to its use on huge data. The following section proposes a synthetic description of three methods, previously proposed in [12-15], and of some results they provide, illustrating the way the grey-level HMT can be used for vessel segmentation, and hopefully justifying its usefulness for many other kinds of medical and non-medical applications.

\section{A few grey-level HMT-based methods}

Vessel segmentation is often applied on angiographic data visualising cerebral or hepatic structures. In such cases, the segmented vessels can be used for planning surgical procedures on the brain or the liver, or to detect and quantify vascular pathologies. The three vessel segmentation methods relying on greylevel HMT discussed in this section, are devoted to such hepatic and cerebral applications. Two versions of the first method [12,13] are designed to automatically recognize a precise part of the hepatic venous tree from CT-scans: the entrance of the portal vein (EPV) of the liver. The second method [13] proposes a segmentation of this whole hepatic venous tree from similar data. The third one $[14,15]$ enables to segment both venous and arterial structures from MRA of the brain.

In the sequel, the considered 3D angiographic images, denoted by $F$, will be considered as functions $E \rightarrow T$ with $E=[0, \operatorname{dim} X-1] \times[0, \operatorname{dim} Y-1] \times$ $[0, \operatorname{dim} Z-1] \subset \mathbf{Z}^{3}$ (where $\operatorname{dim} X, \operatorname{dim} Y$, and $\operatorname{dim} Z$ are the dimensions of the image) and $T=[a, b] \subset \mathbf{Z}$, then dealing with the discrete nature of medical imaging data. The structuring functions involved in grey-level HMT, denoted by $V, W$ will then be functions $E \rightarrow \mathbf{Z}$. All notations used hereafter follow those of Part I of this paper [1]. The grey-level HMT operators are also the ones defined in Section 3 of [1].

\subsection{Choice of structuring functions}

The first issue, when using grey-level HMT for segmentation, is to correctly determine the structuring functions enabling to obtain correct results. More especially, the choice of the "shape" of these functions, which means the support $\operatorname{supp}(V)$ of the foreground function $V$ and the dual support $\operatorname{supp}^{*}(W)$ of the background function $W$, is fundamental. The geometric properties of vessels, which can be modelled as elongated structures presenting a globally circular cross-section, can be used to guide this determination. Since the chosen shapes can present degrees of freedom in terms of size and of orientation, two strategies can then be considered.

The first one consists in determining a fixed shape for the structuring functions. It is applicable when the searched structures present few variations between different patients. However it can sometimes be useful to cope with these variations when using fixed shape structuring functions. To match noisy objects or structures with slightly different shapes the usual method then consists in relaxing the constraints imposed by the structuring functions. Several strategies have been proposed in the literature:

- The erosion of both structuring function supports (increasing the "don't care space" [20]). The main drawback of this method is the possible alteration of the boundaries of structuring function supports: important characteristics of the searched shape may then be removed.

- Using the HMT with rank-order operators [16,2123], which is an efficient way to detect shapes slightly altered by noise or imperfections.

- The subsampling or decimation of the structuring function supports in a regular manner, using Bloomberg's method [21,24].

It has been experimentally observed that the decimation method provides similar results as those obtained from HMT with rank-order operators, with the advantage that decimation reduces the computational complexity.

Using a fixed shape for the structuring functions is the strategy considered in $[12,13]$ where the structuring function supports are chosen according to a priori anatomical knowledge. Indeed, the purpose is to segment a particular structure: the EPV of the liver, 

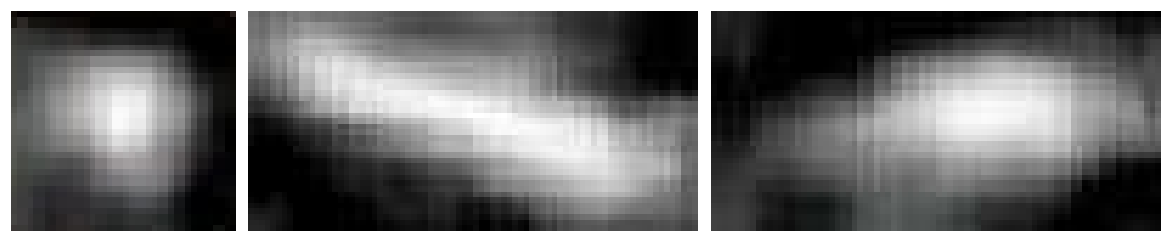

Fig. 2. Mean shape of the EPV, computed from a 18 case training base. Left: sagittal view. Middle: coronal view. Right: axial view.

which presents few anatomical variations. To automatically detect the EPV from CT-scans of the liver, two different versions of the methods are used. In [12], the shapes of the structuring functions are chosen according to the mean shape of the EPV calculated on a training base of images (see Fig. 2). The shapes deduced from this mean representation model (illustrated in Fig. 3) are the following: $\operatorname{supp}(V)$ is a cylinder parallel to the coronal plane and presenting an angle of $\pi / 4$ with the axial plane, while $\operatorname{supp}^{*}(W)$ is a hollow cylinder with the same axis as $\operatorname{supp}(V)$ and with higher radius. A grey-level HMT (based on Soille's integral interval operator $\left.I K_{V, W}\right)$ is then used with these structuring functions, enabling to highlight all the candidates structures. A next step consists in eliminating the false-positives based on some heuristics on the localisation and the size of EPV. In [13], another version of such a strategy is used. A first step detects the superior mesenteric vein (SMV) which is a structure connected to the EPV, while a second step detects the EPV itself. The shape of the SMV is always similar to an elongated vertical structure, justifying the choice of a vertical cylinder for $\operatorname{supp}(V)$, and of a hollow cylinder with the same axis for $\operatorname{supp}^{*}(W)$. For detecting the EPV, a horizontal cylinder is chosen for $\operatorname{supp}(V)$, while a set of three hollow cylinders of same axis but with different sizes is considered for $\operatorname{supp}^{*}(W)$. This enables to deal with the variability of the EPV in terms of size (it has to be noticed that the EPV presents few variations between patients in terms of orientation). These structuring functions are illustrated in Fig. 4. Once both grey-level HMTs have been performed, the intersection between the two resulting images permits keeping only the points belonging to the EPV and SMV, and hence eliminating false positives. A morphological reconstruction starting from these points can then be performed to reconstruct the connected component corresponding to the EPV.

The second strategy for determining the structuring
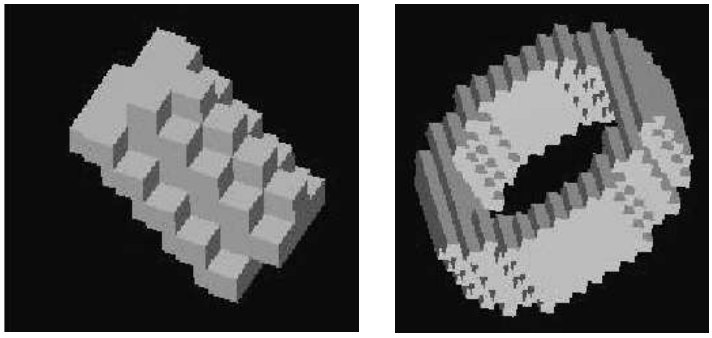

Fig. 3. Shape of the structuring functions used in [12]. Left: foreground element $(\operatorname{supp}(V))$. Right: background element $\left(\operatorname{supp}^{*}(W)\right)$.

functions consists in considering a large set of elements, each one differing in terms of size and orientation. This approach is the one proposed in $[14,15]$, where it is assumed that any size and any orientation can lead to the creation of structuring functions which may be used during the HMT segmentation. Here $\operatorname{supp}(V)$ is chosen as being a discrete sphere. The use of the discrete version of an isotropic shape is justified by the presence of tortuous arterial vessels which could hardly be detected by elongated structures such as ellipsoids. The background shape $\operatorname{supp}^{*}(W)$ is a set of points regularly sampled on a discrete circle with the same centre as $\operatorname{supp}(V)$. The use of a subset of a discrete circle instead of a whole one enables to obtain more robust results at positions such as bifurcations, where the vessels present a non circular cross section. These discrete shapes and their theoretical continuous versions are illustrated in Fig. 5. They present different properties linked to two degrees of freedom in terms of size (one for $\operatorname{supp}(V)$ and one for $\left.\operatorname{supp}^{*}(W)\right)$ and two degrees of freedom in terms of orientation (for $\left.\operatorname{supp}^{*}(W)\right)$. Then, this second strategy leads no longer to a unique element, but to a large family of structuring functions varying according to the different parameters. A subset of this family of elements is illustrated in Fig. 6.

The last parameter which has to be determined is the intensity of the structuring functions, i.e. the respective 


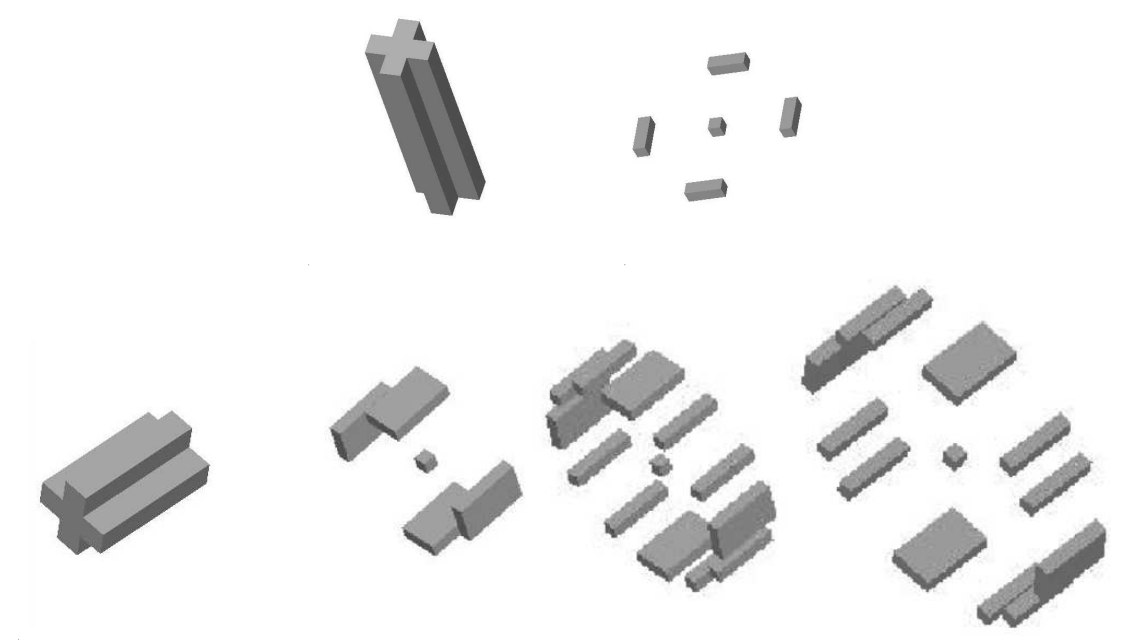

Fig. 4. Shape of the structuring functions used in [13]. First row: structuring functions used for detecting the SMV. Left: foreground element $(\operatorname{supp}(V))$. Right: background element $\left(\operatorname{supp}^{*}(W)\right)$. The central point represents the origin and does not belong to supp* $(W)$. Second row: structuring functions used for detecting the EPV. From left to right: foreground element $(\operatorname{supp}(V))$, background elements $(\operatorname{supp} *(W))$. The central point represents the origin and does not belong to $\operatorname{supp}^{*}(W)$.
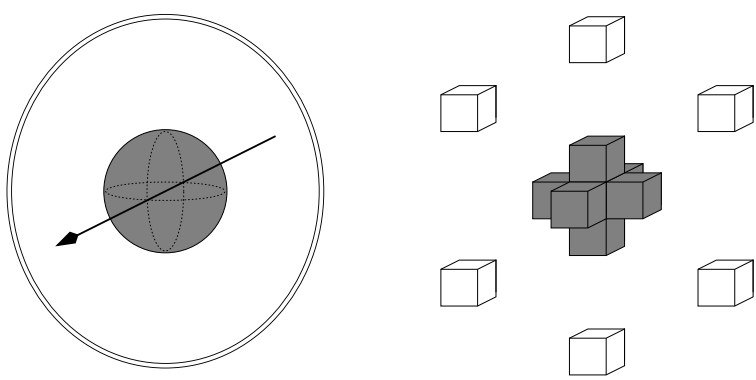

Fig. 5. Shape of the structuring functions used in $[14,15]$. Left: theoretical continuous shapes. Right: real discrete ones. The foreground elements $(\operatorname{supp}(V))$ are represented in dark grey, while the background ones $\left(\operatorname{supp}^{*}(W)\right)$ are represented in white.

values of $V$ and $W$ on $\operatorname{supp}(V)$ and $\operatorname{supp}^{*}(W)$. In [1215], $V$ and $W$ are assumed to present each a constant value on $\operatorname{supp}(V)$ and $\operatorname{supp}^{*}(W)$. These two values are chosen in such a way that the smallest positive difference between image values on $\operatorname{supp}(V)$ and on $\operatorname{supp}^{*}(W)$ leads to a positive response. Thus we take - $V=C_{\text {supp }(V), 0}$, that is $V(x)=0$ for all $x \in \operatorname{supp}(V)$, and

- $W=C_{\text {supp }^{*}(W),-1}^{*}$, that is $W(y)=-1$ for all $y \in$ $\operatorname{supp}^{*}(W)$, with the fitting $H_{V, W}$, or equivalently ([1])

- $W=C_{\text {supp }^{*}(W), 0}^{*}$, that is $W(y)=0$ for all $y \in$ $\operatorname{supp}^{*}(W)$, with the fitting $K_{V, W}$.

This enables the methods to rely more strongly on

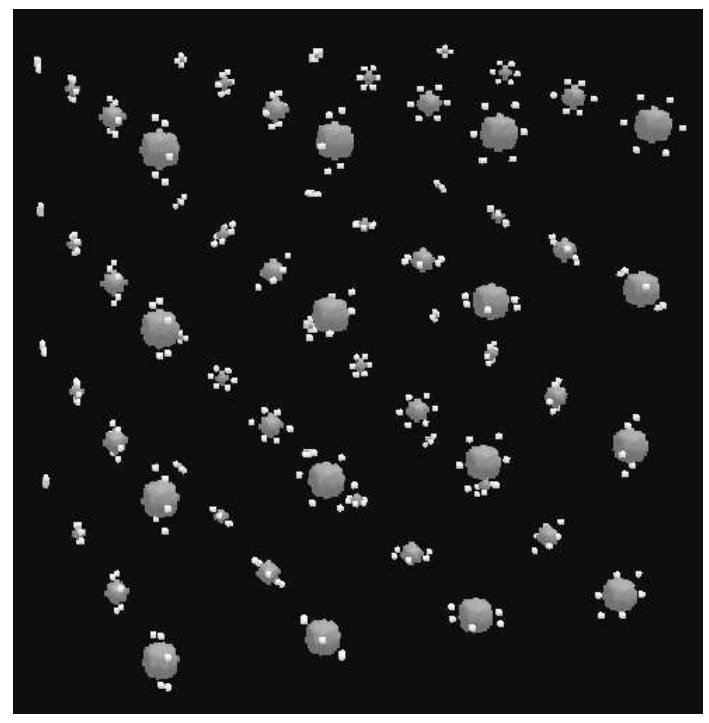

Fig. 6. Subset of the possible structuring function supports used in $[14,15]$. The foreground elements $(\operatorname{supp}(V))$ are represented in dark grey, while the background ones $\left(\operatorname{supp}^{*}(W)\right)$ are represented in white. They present specific properties in terms of size $(\operatorname{supp}(V)$, $\left.\operatorname{supp}^{*}(W)\right)$ and of orientation $\left(\operatorname{supp}^{*}(W)\right)$.

shape properties of the searched vessels than on the possibly low or inhomogeneous signal of the flowing blood, caused by noise or artifacts.

Note that in the particular case of the integral val- 
uation, it is sometimes possible to derive the interval operator from the corresponding one with flat structuring functions. Consider two structuring elements $A, B \in \mathcal{P}(E)$, the structuring functions $V=C_{A, a}$ and $W=C_{B, b}^{*}$ (for $a, b \in T^{\prime}$ ), and the associated flat structuring functions $V^{\prime}=C_{A, 0}$ and $W^{\prime}=C_{B, 0}^{*}$. Then for any function $F \in T^{E}$ and $p \in E$, we have

$$
I H_{V^{\prime}, W^{\prime}}(F)(p)=\max [(F \ominus A)(p)-(F \oplus \check{B})(p), 0]
$$

and

$$
\begin{aligned}
& I H_{V, W}(F)(p)= \\
& \quad \max [(F \ominus A)(p)-(F \oplus \check{B})(p)-a+b, 0] .
\end{aligned}
$$

If $a \geq b$ we get:

- IH $H_{V, W}(F)(p)=\max \left[I H_{V^{\prime}, W^{\prime}}(F)(p)-a+b, 0\right]$, and similarly

- $I K_{V, W}(F)(p)=\max \left[I K_{V^{\prime}, W^{\prime}}(F)(p)-a+b, 0\right]$.

In other words, when $a \geq b$, from the integral valuation associated to the fitting obtained with two flat structuring functions $V^{\prime}$ and $W^{\prime}$, we can derive the integral valuation associated to the fitting obtained with non-flat structuring functions $V=C_{A, a}$ and $W=C_{B, b}^{*}$.

If $a<b$ this relation does not hold anymore, and we can only say that

$$
\begin{aligned}
I H_{V^{\prime}, W^{\prime}}(F)(p)>0 & \Longrightarrow \\
I H_{V, W}(F)(p) & =I H_{V^{\prime}, W^{\prime}}(F)(p)-a+b,
\end{aligned}
$$

and similarly for $I K_{V, W}(F)(p)$. Indeed, the integral valuation "loses" all negative differences $(F \ominus A)(p)-$ $(F \oplus \breve{B})(p)$, whose valuation is set to 0 .

From an algorithmic point of view, in order to compute multiple grey-level hit-or-miss transforms (with integral valuation) with structuring functions $V=C_{A, a}$ and $W=C_{B, b}^{*}$ for increasing $t=a-b \geq 0,(a, b \in \mathbf{Z})$, it is sufficient to compute only one HMT with $V=C_{A, 0}$ and $W=C_{B, 0}^{*}$. Other HMTs are obtained by subtracting $t$ to each point. Note that $t=a-b<0$ is not a problem in practice: one can for example define another integral valuation taking all possible (i.e. positive and negative) values.

\subsection{A few remarks about the flat/non-flat structuring functions}

The vessel segmentation methods described in this paper only use structuring functions with constant grey-levels, flat or not (those structuring functions being cylinders $C_{A, t}$ ). Theoretically, it is however possible to design non-flat structuring elements with non-constant grey-levels. Such structuring functions are more constrained, enabling to segment precise structures according to their shape but also to precise local intensity properties. In the case of angiographic data analysis, a few situations could justify the development of strategies using non-flat structuring function with non-constant grey-levels. Such situations are related to vascular (or more generally anatomical) objects presenting characteristic textures.

A first case is linked to the well known partial volume effect, leading to smooth transitions between structures, in the regions where voxels contain several different tissues. A HMT using grey-level structuring elements to detect this type of transitions (relying on a family of structuring functions whose slope is the same as the transition between tissues) could be considered in this kind of situation. However in such a case, a flat structuring element with an integral valuation could also be used to detect the local variations of the function.

A second case is linked to a precise category of MRA, called Phase-Contrast MRA, which detects the flowing blood movement, and more especially the phase shift of moving spins in the blood. The spin movement being cyclic, signal artifacts can appear in the resulting angiographic data. They generally correspond to a signal decrease at the center of the vessels, where the blood signal should reach its maximal value. These aliasing artifacts lead to a blood signal locally similar to a high intensity ring surrounding a lower intensity area (which can sometimes be even lower than the background signal). A HMT using grey-level structuring elements could then enable the detection of blood signal, by considering a structuring function correctly fitting this kind of partial signal. However, the definition of such functions would first require to model the physical phenomena leading to these artifacts, and to consider several acquisition parameters which are not necessarily available. Moreover, even in this case, the use of a flat structuring function with well-chosen shapes (for example two hollow cylinders of same axis, modeling the vessel and the background) could probably also deal with this kind of artifacts.

Finally, in practical cases (where the purpose is 
generally to characterize structures from their shape by imposing a constraint on the difference of contrast between the object and a particular neighborhood), flat structuring elements are generally sufficient. The determination of situations where the use of non-flat structuring elements could be justified is not an easy task. This is a general question in mathematical morphology, as erosions and dilations using nonflat and non-constant structuring functions have seen little practical use for now. This is the case when one wants to impose not only constraints on the contrast between an object and its neighborhood, but also a particular ordering of the grey-levels inside the shape or its neighborhood. However the main difficulty remains the choice of grey-levels in order to deal with the variability of real image objects. In such situations, indeed, one has to face with "horizontal" variability (variation of "shape") along with "vertical" variability (variation of scene illumination, scaling of intensities), thus incresing the number of parameters of the problem.

\subsection{Algorithmic process}

As previously stated, the grey-level HMT can essentially be used in two main ways: in a classical filtering process, or as part of heuristic criteria for guidance of iterative segmentation processes.

\subsubsection{Filtering segmentation}

Filtering segmentation consists in applying a fitting which associates to $F$ a set of points $(p, t) \in E \times T^{\prime}$ for which $V_{(p, t)}$ and $W_{(p, t)}$ have some relation to $F$. Such an exhaustive strategy implies that all the considered pairs of structuring functions $(V, W)$ have to be tested on each point of the processed image $F$. This leads to complexities:

- $O_{1}=O(\operatorname{card}(E))=O(\operatorname{dim} X \cdot \operatorname{dim} Y \cdot \operatorname{dim} Z)$, for the number of points where HMTs are applied;

- $O_{2}=O(\operatorname{card}(\{V, W\}))$, for the number of applied HMTs on each point (where card $(\{V, W\})$ stands for the number of applicable pairs of elements $V$ and $W)$;

- $O_{3}=O(\operatorname{card}(V) \cdot \operatorname{card}(W))$, for the application of one HMT on one point of $F$.

The global complexity of a filtering vessel segmentation $O=O_{1} \cdot O_{2} \cdot O_{3}$ can then become prohibitive if an exhaustive approach is considered. (Note however that the complexity $O_{1} . O_{3}$ of a single HMT can be reduced when the structuring elements $V$ and $W$ satisfy some conditions, see [25,26] and [16, p. 81].)

In [12], the processed images are subsampled to reduce the complexity $O_{1}$. Moreover, since a fixed number of structuring functions are considered, the $\mathrm{O}_{2}$ complexity is equal to $O(1)$. Finally, $O_{3}$ is also reduced by simplifying the structuring function supports according to Bloomberg's method [24] which consists in removing points in a regular manner (see Fig. 7). Note that in this case the primary goal of this simplification (or decimation) is not the reduction of the complexity, but rather the relaxing of the constraints imposed by the structuring functions (see Subsection 3.1). Here the complexity reduction then appears as an "interesting" side effect of decimation.

The fitting step in $[12,13]$ is $K_{V, W}$ (following Soille's approach), while the valuation is the binary mask one $M$. The result $M K_{V, W}$ is then intersected with the original image to keep the original grey-levels, finally leading to the following filtering segmentation formula:

$$
\begin{aligned}
& F \wedge M K_{V, W}(F): \\
& \qquad p \mapsto \begin{cases}F(p) \text { if } \exists t \in T^{\prime}, V_{(p, t)} \leq F \ll W_{(p, t)}, \\
-\infty \text { otherwise } .\end{cases}
\end{aligned}
$$
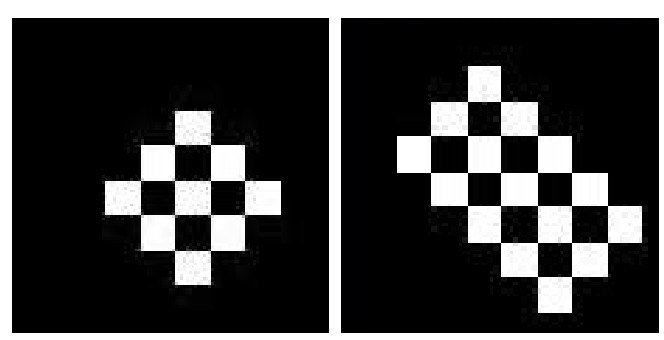

Fig. 7. Structuring function support subsampled on a regular grid. Left: sagittal plane. Right: axial plane.

In $[14,15]$, the number of pairs of structuring functions used for HMT (i.e. the $O_{1} . O_{2}$ complexity) is $O\left(\operatorname{dimX} \cdot \operatorname{dim} Y \cdot \operatorname{dim} Z \cdot R_{V} \cdot R_{W} \cdot \theta_{W} \cdot \phi_{W}\right)$, where $R_{V}, R_{W}, \theta_{W}, \phi_{W}$ are the radii of $\operatorname{supp}(V)$ and $\operatorname{supp}^{*}(W)$ and the orientations of $\operatorname{supp}^{*}(W)$ in a spherical frame (i.e. the degrees of freedom of parameters of the structuring function supports), respectively. Since this complexity forbids to obtain results without expensive computation time, the proposed way to reduce 
$O_{1} . O_{2}$ consists in using an atlas, which is a function $\mathcal{A}$ defined on $E$ and indicating for each point $p \in E$ if a grey-level HMT has to be applied on $p$ or not, and what are the subsets of parameters $R_{V}, R_{W}, \theta_{W}$, and $\phi_{W}$ which should be considered to define the different structuring functions $V$ and $W$ applicable on $p$. In the sequel, the notation $(V, W) \in \mathcal{A}(p)$ will be used to indicate that the pair of structuring functions $(V, W)$ presents correct properties with respect to $\mathcal{A}$, and can then be applied on point $p$. The $O_{1} . O_{2}$ complexity is then no longer equal to the previously given formula, but to $O\left(\sum_{p \in E} \operatorname{card}(A(p))\right)$. In the case of cerebral MRA segmentation, such an atlas $\mathcal{A}$ (which can be generated by an anatomical property extraction process $[15,27])$ enables to dramatically reduce the number of HMT applications without altering the quality of the results. The fitting step of the HMT segmentation proposed in $[14,15]$ (based on Ronse's $\left.H_{V, W}\right)$ can then be defined by:

$$
\begin{aligned}
H_{\mathcal{A}}(F)= & \left\{(p, t) \in E \times T^{\prime} \mid\right. \\
& \left.\exists(V, W) \in \mathcal{A}(p), V_{(p, t)} \leq F \leq W_{(p, t)}\right\} .
\end{aligned}
$$

The chosen valuation used for this segmentation method is the binary one $B$. It has to be noticed that since the main purpose of this method is to determine a binary image of the whole vascular structures, it is necessary to finally provide a volumic object. As the previously defined set represents a "skeletal" segmentation only visualising the centre points of the vessels, a volumic segmentation can be obtained by dilating each segmented point with the foreground element $\operatorname{supp}(V)$ which enabled its detection. The final segmentation can then be defined by:

$$
\begin{aligned}
& \bigcup_{p \in E}\left\{\operatorname{supp}(V) \oplus\{p\} \mid \exists t \in T^{\prime}, \exists W,\right. \\
& \left.\quad(V, W) \in \mathcal{A}(p), V_{(p, t)} \leq F \leq W_{(p, t)}\right\} .
\end{aligned}
$$

\subsubsection{Heuristic criteria for region-growing segmentation}

While filtering methods tend to apply HMT on each point of the processed image, the use of HMT as a heuristic criterion is quite different, as it consists in applying it only on candidate points during a vessel segmentation process. The use of such criteria is generally considered in iterative segmentation processes such as region-growing or vessel-tracking ones. The application proposed hereafter is based on region-growing and corresponds to the method described in [13].

Region-growing segmentation consists in starting from a seed point, or a seed region which is assumed to belong to the searched object. It then iteratively adds points of the image to this seed, until obtaining the whole structure to be segmented. As a consequence, a region-growing segmentation only requires two elements: the seed $S \subset E$ and a heuristic criterion $C$ (which can be seen as a Boolean function defined on $E$ and depending on several parameters) indicating if a candidate point can be added to the currently segmented object. The region-growing segmentation of an image $F$ can then be formalised as the construction of a sequence $\left\{S_{k}\right\}_{k \in \mathbf{N}}$ :

$$
\begin{aligned}
& S_{0}=S, \\
& \forall k \geq 0, S_{k+1}= \begin{cases}S_{k} \cup\{p\} & \text { if } \exists p \in N\left(S_{k}\right), \\
& C\left(E, S_{k}, p, \ldots\right)=\text { true }, \\
S_{k} & \text { otherwise . }\end{cases}
\end{aligned}
$$

where $N\left(S_{k}\right)$ represents the set of neighbour pixels of $S_{k}$ according to a chosen connexity. The obtained segmentation is then given by:

$S=\bigcup_{k=0}^{\infty} S_{k}=\lim _{k} S_{k}$.

The sequence $\left\{S_{k}\right\}_{k \in \mathbf{N}}$ being increasing, the segmentation process necessarily ends for finite images.

A heuristic criterion based on shape and contrast is used in [13] to characterize bright tubular structures. It is assumed that bright pixels surrounded by a ring of darker ones are likely to be included in a vascular network. This is the principle of the criterion used to segment the vascular network of the liver. Regiongrowing segmentation is performed by starting from the EPV, this seed being preliminarily detected by the previously described filtering method. The criterion used for the region-growing segmentation can be expressed as:

$C(F, p)=\left\{\begin{array}{l}\text { true if } \max _{i=1}^{3}\left[S K_{O, R_{i}}(F)\right](p)>0, \\ \text { false otherwise },\end{array}\right.$

or: 
$C(F, p)=\bigvee_{i=1}^{3}\left(\left[S K_{O, R_{i}}(F)\right](p)>0\right)$,

where $O=i_{0,0}\left(i_{p, t}\right.$ being the impulse function) is the structuring element only composed of the origin and $R_{i}(i=1$ to 3$)$ are structuring elements used to constrain the point $p$ to belong to a tubular structure. Such structuring elements $R_{i}$ are three orthogonal discrete rings of radius $r$ and thickness $t$, parallel to the axial, coronal and sagittal planes (see Fig. 8). These two parameters can be used to control the segmentation result by determining the size of the branches detected during the region-growing (see Fig. 9 and 10).

Note that in this case, as the criterion $C$ does not vary during the propagation (i.e. it remains the same for all $p \in E$ ), the result of the region-growing algorithm is equivalent to applying the criterion $C$ to all points and keeping in the resulting binary image the connected component containing the seed $S$ (using the same connexity as for the neighborhood function $N$ ). However from an algorithmic point of view it is more interesting to use the region-growing formulation since it reduces the complexity: the HMTs are computed only for the points included in the final result (for which the criterion $C$ is true) and their neighbors (for which the criterion $C$ is false).

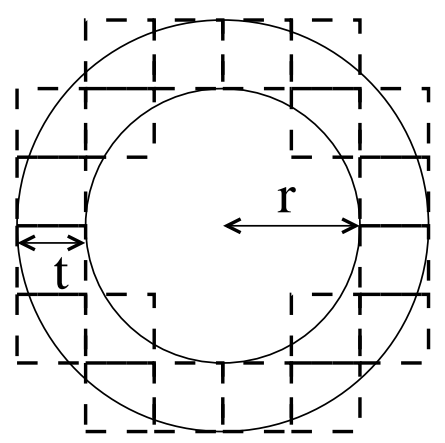

Fig. 8. Discrete ring of radius $r$ and thickness $t$, used to generate structuring elements $R_{i}$ involved in the region-growing segmentation process of [13].

\subsection{Results}

The segmentation methods devoted to the EPV, described in [12,13], have been applied on a 16 case dataset. The detection of the EPV was successful for all images, leading to a detection rate of $100 \%$. This segmentation enables to obtain a robust seed (robust in the sense that small variations in seeds lead to similar results) used in a second step to segment the hepatic portal network using a region-growing algorithm (described above and in [13]). As illustrated in Fig. 11, the process provides visually satisfying results. The number of segmented branches and the global quality of the segmentation are variable between the cases, depending on the quality of the CT-scan acquisition. Indeed, the highlighting of the vascular network obtained from the contrast medium varies with the acquisition time. This leads to a great variability between acquisitions. It should also be noticed that the main purpose of these acquisitions remains the highlighting of the liver, and not the hepatic network. In such conditions, the obtained results are quite satisfactory since the maximal information available in the images is extracted. A comparative study has been performed between this method and a previous one described in [28-32]. This other method uses also a region-growing algorithm but involves a criterion based on a fixed threshold. It has been experimentally observed, on the image dataset, that the threshold was either set too high, leading to poor results (with very few branches), either set too low, leading to an erroneous propagation extending into neighbouring organs. The better results obtained from our method are mainly justified by the criterion used for segmentation, which relies no longer on threshold information as in [28-32], but on local contrast, which seems actually more robust and accurate.

The segmentation method proposed in $[14,15]$ has been applied on a dataset composed of 30 cerebral phase-contrast MRA (left part of Fig. 1), presenting dimensions varying from $256^{2} \times 150$ to $256^{2} \times 180$ voxels and millimetric resolution. The obtained binary segmentations have been compared to those provided by another vessel segmentation process [33], emphasising imperfect but already promising results in terms of false negatives, and quite satisfying ones in terms of false positives. By comparison with other brain vessel segmentation strategies, this method presents specific properties which are strongly linked to the use of grey-level HMT. Indeed, since it relies much more on shape properties of the vessels than on intensity properties of the flowing blood, this segmentation process is less sensitive to vascular signal imperfections which can happen in MRA, such as aliasing artifacts, signal 

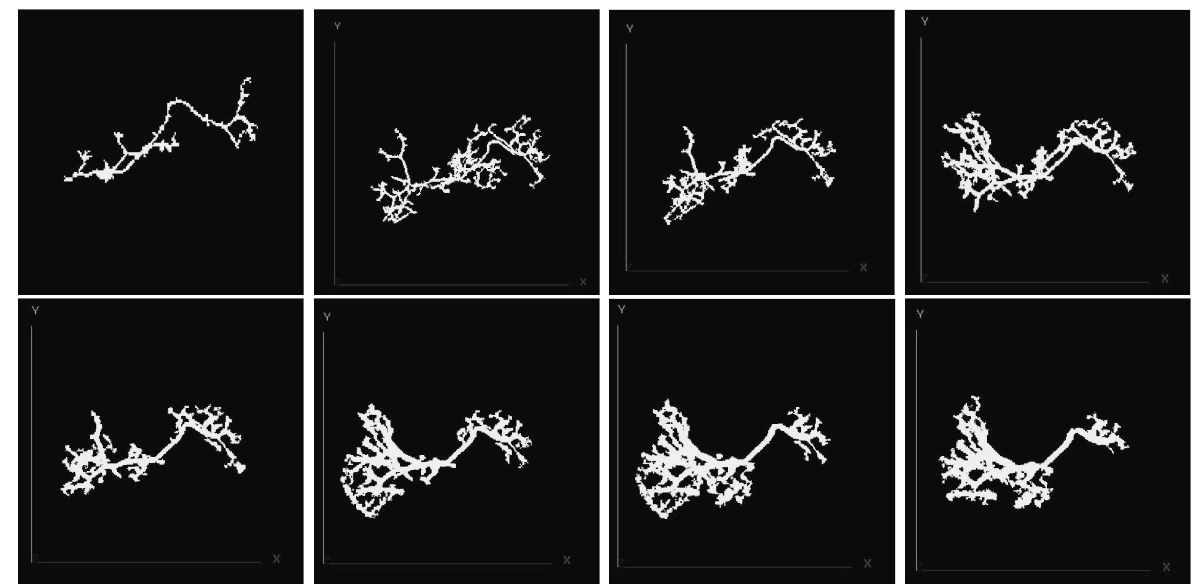

Fig. 9. Segmentation result of the region-growing segmentation of [13], using the criterion $C$ and $R_{i}$ elements of fixed thickness $t=1 \mathrm{~mm}$ and increasing radii (top left to bottom right: $r=2 \mathrm{~mm}$ to $r=9 \mathrm{~mm}$ ).
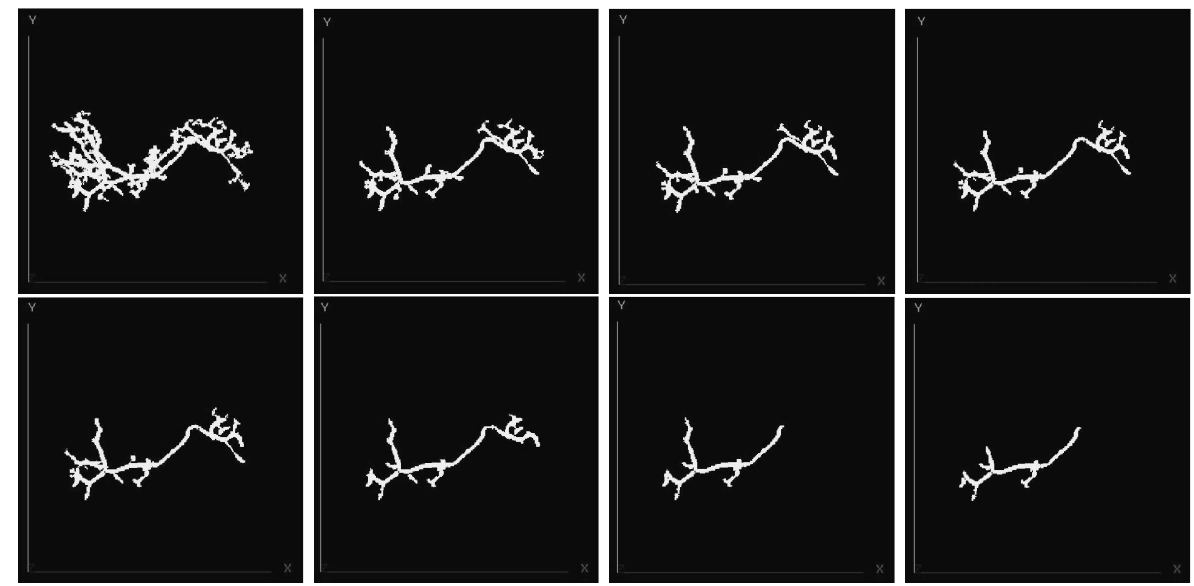

Fig. 10. Segmentation result of the region-growing segmentation of [13], using the criterion $C$ and $R_{i}$ elements of fixed radius $r=5$ mm and increasing thickness (top left to bottom right: $t=2 \mathrm{~mm}$ to $t=9 \mathrm{~mm}$ ).

inhomogeneities or signal loss due to flow turbulence, or patient movement artifacts. This shape-based behaviour is a major advantage for vessel segmentation applications which do not require to mainly consider blood intensity, as neurosurgery planning or vascular landmark detection for functional analysis. A segmented vascular tree obtained from this dataset, visualising both arterial and venous structures of the brain, is illustrated in Fig. 12.

Finally, the different methods proposed in this section and the results they provide for segmentation of hepatic and cerebral vessels tend to demonstrate the usefulness and efficiency of the grey-level HMT in the field of angiographic data analysis, but also in the more general one of medical image analysis. Indeed, the high adaptiveness of this operator concerning shape and intensity, and its ability to detect structures by considering their properties but also the properties of their neighbourhood, constitute real advantages for applications related to medical images, where highlevel a priori anatomical knowledge about the studied structures can often be used to guide image processing tools. 


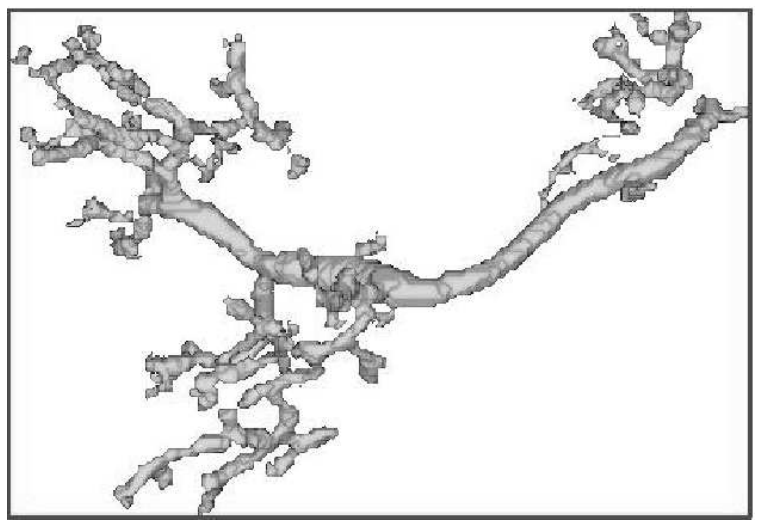

Fig. 11. 3D surface rendering visualisation of the portal network structures segmented from CT-data of the liver.

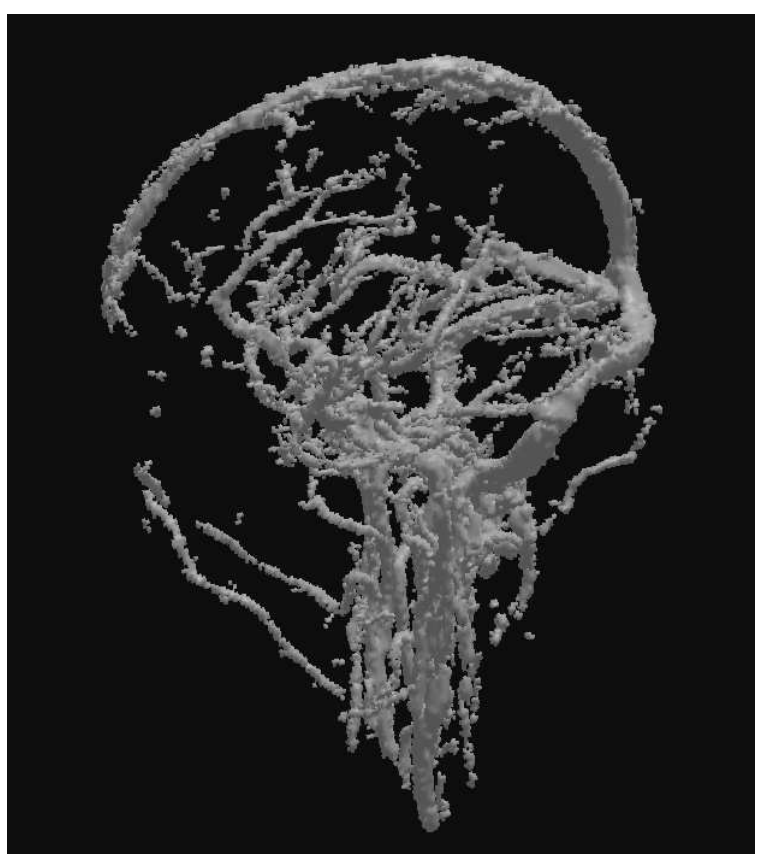

Fig. 12. 3D surface rendering visualisation of cerebral vascular structures segmented from a phase-contrast MRA of the brain.

\section{Conclusion}

After the unified theory proposed in the first part of this paper [1], this second part has presented applicative aspects of grey-level HMT. Several vessel segmentation methods devoted to 3D angiographic data have been described. The accuracy of the results they provide on datasets prove that the underuse of grey-level
HMT is probably unjustified in the fied of medical image analysis, and more globally in the field of image processing. The different discussions in the previous sections also emphasize several ways enabling to easily involve grey-level HMT in various general segmentation strategies (region-growing, filtering, ...), but also to reduce the computational complexity of HMTbased methods by considering various heuristics. This should convince the reader of the wide applicability of these operators in grey-level image processing.

In the field of vessel segmentation from 3D data, further works based on grey-level HMT will now consist in improving some of the methods described in this paper by increasing the adaptiveness of the structuring functions used for HMT, in order to improve the segmentation accuracy and robustness.

\section{Acknowledgement}

The authors thank Joseph Baruthio, Jean-Paul Armspach, Claude Maillot and Luc Soler for their contribution to the experimental works of this paper. Anatomical images of the head were provided by the radiology service of the Strasbourg University Hospital, while those of the liver came from the Research Institute against Cancer of the Digestive System in Strasbourg. They are grateful to the referee for constructive suggestions.

\section{References}

[1] B. Naegel, N. Passat, C. Ronse, Grey-level hit-or-miss transforms - Part I: Unified theory, Submitted for publication.

[2] R. Schwartz, K. Jones, D. Chernoff, S. Mukherji, R. Khorasani, H. Tice, R. Kikinis, S. Hooton, P. Stieg, J. Polak, Common carotid artery bifurcation: Evaluation with spiral CT, Radiology 185 (2) (1992) 513-519.

[3] C. Dumoulin, H. Hart, Magnetic resonance angiography, Radiology 161 (3) (1986) 717-720.

[4] M. Prince, E. Yucel, J. Kaufman, D. Harrisson, S. Geller, Dynamic gadolinium-enhanced three-dimensional abdominal MR arteriography, Journal of Magnetic Resonance Imaging 3 (6) (1993) 877-881.

[5] C. Dumoulin, S. Souza, M. Walker, W. Wagle, Threedimensional phase contrast angiography, Magnetic Resonance in Medicine 9 (1) (1989) 139-149.

[6] F. Wehrli, A. Shimakawa, G. Gullberg, J. McFall, Time of flight MR flow imaging: Selective saturation recovery with gradient refocusing, Radiology 160 (3) (1986) 781-785. 
[7] Y. Sun, D. Parker, Performance analysis of maximum intensity projection for display of MRA data, IEEE Transactions on Medical Imaging 18 (12) (1999) 1154-1169.

[8] K. Bühler, P. Felkel, A. L. Cruz, Geometric methods for vessel visualization and quantification - A survey, Technical Report VRVis-2002-035, VRVis Center, Vienna (2002).

[9] P. Felkel, R. Wegenkittl, A. Kanitsar, Vessel tracking in peripheral CTA datasets - An overview, in: SCCG'01 17th Spring Conference on Computer Graphics, Proceedings, IEEE, Budmerice, Slovakia, 2001, pp. 232-239.

[10] C. Kirbas, F. Quek, A review of vessel extraction techniques and algorithms, ACM Computing Surveys 36 (2) (2004) 81121.

[11] J. Suri, K. Liu, L. Reden, S. Laxminarayan, A review on MR vascular image processing: Skeleton versus nonskeleton approaches: Part II, IEEE Transactions on Information Technology in Biomedicine 6 (4) (2002) 338-350.

[12] B. Naegel, C. Ronse, L. Soler, Segmentation automatique de l'entrée de la veine porte dans le foie par application de la transformée en tout-ou-rien en niveaux de gris, in: ORASIS 2003, 9e congrès, Proceedings, Gérardmer, France, 2003, pp. 33-37.

[13] B. Naegel, C. Ronse, L. Soler, Using grey-scale hit-ormiss transform for segmenting the portal network of the liver, in: C. Ronse, L. Najman, E. Decencière (Eds.), Mathematical Morphology: 40 years on. Proceedings of the 7th International Symposium on Mathematical Morphology, Vol. 30 of Computational Imaging and Vision, Springer SBM, Paris, France, 2005, pp. 429-440.

[14] N. Passat, C. Ronse, J. Baruthio, J.-P. Armspach, Automatic parameterization of grey-level hit-or-miss operators for brain vessel segmentation, in: ICASSP'05 - 30th International Conference on Acoustics, Speech, and Signal Processing, Proceedings, Vol. 2, IEEE, Philadelphia, Pennsylvania, USA, 2005, pp. 737-740.

[15] N. Passat, C. Ronse, J. Baruthio, J.-P. Armspach, C. Maillot, Magnetic resonance angiography: From anatomical knowledge modeling to vessel segmentation, Medical Image Analysis 10 (2) (2006) 259-274.

[16] P. Soille, Morphological Image Analysis: Principles and Applications, 2nd Edition, Springer-Verlag Berlin Heidelberg, 2003.

[17] J. Seneschal, C. Fournier, C. Barat, T. Fournel, Segmentation 3D d'hologrammes numériques de particules par palpage morphologique : application à l'analyse d'un jet diesel hautepression, in: GRETSI'03 - 19e colloque GRETSI sur le traitement du signal et des images, Proceedings, Vol. 2, Paris, France, 2003, pp. 311-315.

[18] C. Fournier, C. Barat, C. Ducottet, T. Fournel, Digital holography applied to PIV hologram reconstruction and selection of a cloud of particles in the reconstructed volume, in: PSFVIP4 - 4th Pacific Symposium on Flow Visualization and Image Processing, Proceedings, Chamonix, France, 2003, p. F4093.

[19] C. Barat, C. Ducottet, M. Jourlin, Line pattern segmentation using morphological probing, in: S. Loncaric, A. Neri, H. Babic (Eds.), ISPA'05 - 3rd International Symposium on Image and Signal Processing and Analysis, Proceedings, Vol. 1, IEEE, Rome, Italy, 2003, pp. 417-422.

[20] D. Bloomberg, Multiresolution morphological approach to document image analysis, in: ICDAR'91 - 1st International Conference on Document Analysis and Recognition, Proceedings, Saint-Malo, France, 1991, pp. 963-971.

[21] D. Bloomberg, P. Maragos, Generalized hit-miss operations, in: P. Gader (Ed.), Image Algebra and Morphological Image Processing 1998, Proceedings, Vol. 1350, SPIE, San Diego, California, USA, 1990, pp. 116-128.

[22] P. Soille, On morphological operators based on rank filters, Pattern Recognition 35 (2002) 527-535.

[23] C. Ronse, A lattice-theoretical morphological view on template extraction in images, Journal of Visual Communication and Image Representation 7 (3) (1996) 273 295.

[24] D. Bloomberg, L. Vincent, Pattern matching using the blur hit-miss transform, Journal of Electronic Imaging 9 (2) (2000) $140-150$.

[25] M. van Herk, A fast algorithm for local minimum and maximum filters on rectangular and octogonal kernels, Pattern Recognition Letters 13 (7) (1992) 517-521.

[26] M. Van Droogenbroeck, H. Talbot, Fast computation of morphological operations with arbitrary structuring elements, Pattern Recognition Letters 17 (14) (1996) 1451-1460.

[27] N. Passat, C. Ronse, J. Baruthio, J.-P. Armspach, C. Maillot, Cerebral vascular atlas generation for anatomical knowledge modeling and segmentation purpose, in: C. Schmid, S. Soatto, C. Tomasi (Eds.), Computer Vision and Pattern Recognition CVPR'05, 23rd International Conference, Proceedings, Vol. 2, IEEE, San Diego, California, USA, 2005, pp. 331-337.

[28] D. Selle, B. Preim, A. Schenk, H.-O. Peitgen, Analysis of vasculature for liver surgical planning, IEEE Transactions on Medical Imaging 21 (11) (2002) 1344-1357.

[29] D. Selle, T. Schindewolf, C. Evertsz, H. Peitgen, Quantitative analysis of CT liver images, in: M. G. K. Doi, H. MacMahon, K. Hoffmann (Eds.), 1st International Workshop on ComputerAided Diagnosis in Medical Imaging, Proceedings, Elsevier, Chicago, Illinois, USA, 1998, pp. 435-444.

[30] D. Selle, W. Spindler, B. Preim, H.-O. Peitgen, Mathematical methods in medical imaging: Analysis of vascular structures for liver surgery planning, in: Engquist, Schmid (Eds.), Mathematics Unlimited 2001 and Beyond, Springer, 2001, pp. 1039-1059.

[31] C. Zahlten, H. Jürgens, C. Evertsz, R. Leppek, H. Peitgen, K. Klose, Portal vein reconstruction based on topology, European Journal of Radiology 19 (2) (1995) 96-100. 
[32] C. Zahlten, H. Jürgens, H. Peitgen, Reconstruction of branching blood vessels from CT-data, in: R. Scateni, J. van Wijk, P. Zanarini (Eds.), Visualization in Scientific Computing'95, 6th Workshop, Proceedings, Eurographics, Chia, Italy, 1995, pp. 41-52.

[33] N. Passat, C. Ronse, J. Baruthio, J.-P. Armspach, C. Maillot, C. Jahn, Region-growing segmentation of brain vessels: An atlas-based automatic approach, Journal of Magnetic Resonance Imaging 21 (6) (2005) 715-725.

\section{Vitae}

\section{Benoît Naegel}

Benoît Naegel was born in 1978. He studied computer science at the Université Louis Pasteur, Strasbourg (Licence, 1998; M.Sc., 2000; Ph.D., 2004). From 2000 to 2004 he worked on medical image segmentation and mathematical morphology at the Research Institute Against Cancer of the Digestive System (IRCAD) under the guidance of Christian Ronse and Luc Soler. He is presently in post-doctoral fellowship as a research assistant at the École d'Ingénieurs de Genève (Switzerland). His research interests include image processing and biomedical applications of mathematical morphology.

\section{Nicolas Passat}

Nicolas Passat was born in 1978. He studied computer science at the Université d'Orléans (Licence, 2000) and the Université Louis Pasteur, Strasbourg (M.Sc., 2002; Ph.D., 2005), specialising in image processing. During his research practice, he worked at the LSIIT and IPB-LNV (Université Louis Pasteur, Strasbourg), on vascular structure segmentation from 3D medical images, under the supervision of Christian Ronse and Joseph Baruthio. He is currently in post-doctoral fellowship at the $\mathrm{A}^{2} \mathrm{SI}$ Laboratory (Université de Marne-la-Vallée). His scientific interests include imaging processing, mathematical morphology, and medical imaging.

\section{Christian Ronse}

Christian Ronse was born in 1954. He studied pure mathematics at the Université Libre de Bruxelles (Li- cence, 1976) and the University of Oxford (M.Sc., 1977; Ph.D., 1979), specialising in group theory. Between 1979 and 1991 he was Member of Scientific Staff at the Philips Research Laboratory Brussels, where he conducted research on combinatorics of switching circuits, feedback shift registers, discrete geometry, image processing, and mathematical morphology. During the academic year 1991-1992 he worked at the Universite Bordeaux-1, where he obtained his Habilitation diploma. Since October 1992, he has been Professor of Computer Science at the Université Louis Pasteur, Strasbourg (promotion to First Class Professorship in 2001), where he contributed to the development of a research group on image analysis, and the teaching of image processing to students at various levels. His scientific interests include imaging theory, mathematical morphology, image segmentation and medical imaging. 